\title{
Funcionalidad familiar y calidad de vida en familias con y sin canes
}

\author{
Yeimi Vanessa Arboleda'; Luisa María Echeverri Vallejo²; \\ Luis Esteban Martínez Lopera ${ }^{3}$; Isabela Márquez ; José Alonso Andrade Salazar ${ }^{5}$
}

RESUMEN Investigación cuantitativa de tipo empírico analítico y de corte transversal, cuyo objetivo es analizar la relación entre funcionalidad familiar y calidad de vida en familias con y sin canes como mascotas, en grupos familiares del dpartamento de Risaralda, a las cuales se aplicará una ficha de caracterización socio-demográfica, el APGAR Familiar de Gabriel Smilkstein (Alegre \& Suárez, 2006) y el test SF-36 de calidad de vida SF-36 (Ware \& Sherbourne, 1992).

Quienes tienen una mascota poseen una buena funcionalidad familiar $(51,3 \%)$ a diferencia de quienes no la tienen $(45,9 \%)$, la disfunción familiar severa $(8,1 \%)$ y moderada $(15,1 \%)$ es mayor cuando no se tiene una mascota. La salud mental y la calidad de vida fueron buenas en el $81,5 \%$ cuando se tiene mascota y en el $70,9 \%$ sin mascota. Se concluye que las mascotas tienen un efecto positivo en la salud mental, las relaciones intrafamiliares y la calidad de vida de las familias, pues mejoran su percepción de bienestar, y la vivencia en aspectos que fortalecen el buen funcionamiento familiar, como adaptación, participación, ganancia, afecto y recursos.

Canes;

Calidad de Vida;

PALABRAS Disfunción Familiar;

CLAVE Familia;

Funcionalidad Familiar.

1 Estudiante de séptimo semestre psicología, Fundación universitaria del Área Andina, email: yarboleda5@estudiantes.areandina.edu.co,

2 Estudiante de séptimo semestre psicología, Fundación universitaria del Área Andina, email: Lecheverri9@estudiantes.areandina.edu.co

3 Estudiante de séptimo semestre psicología, Fundación universitaria del Área Andina, email: Lmartinez147@estudiantes.areandina.edu.co

4 Estudiante de séptimo semestre psicología, Fundación universitaria del Área Andina, email: imarquez@estudiantes.areandina.edu.co

5 Psicólogo. Docente investigador Fundación Universitaria del Área Andina. Docente investigador Universidad de San Buenaventura Medellín extensión Armenia. Magister en Investigación Integrativa. PhD en Pensamiento complejo. Email: jandrade20@areandina.edu.co; jose.andrade@usbmed.edu.co 


\section{Family functionality and quality of life in families with and without dogs}

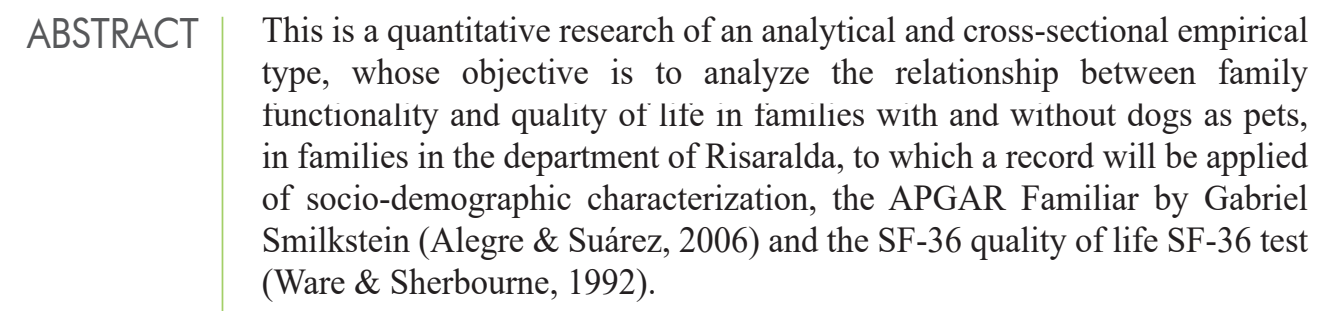

Those who have a pet have good family functionality (51.3\%), unlike those who do not $(45.9 \%)$, severe $(8.1 \%)$ and moderate $(15.1 \%)$ family dysfunction is greater when You don't have a pet. Mental health and quality of life were good in $81.5 \%$ when you have a pet and in $70.9 \%$ without a pet. It is concluded that pets have a positive effect on mental health, intrafamily relationships and the quality of life of families, as they improve their perception of well-being, and the experience of aspects that strengthen family functioning such as: adaptation, participation, gain, affection and resources.

Dogs;

Quality of Life;

KEYWORDS Family dysfunction;

Family;

Family Functionality.. 


\section{INTRODUCCIÓN}

El último estudio nacional en salud mental en Colombia, a través de la Encuesta de Salud Mental del Ministerio de Salud y Protección Social (2015), reveló que existen, según la percepción de la salud mental de los colombianos, cuatro factores que la mejoran en hombres y mujeres: 1) tener buena salud física, comer, dormir, descansar -hombres (47\%) y mujeres (46,9\%); 2) sentirse bien, feliz, en paz con uno mismo: hombres $(36,2 \%)$ y mujeres $(42,2 \%)$; superar dificultades y seguir adelante con ganas: hombres $(35,1 \%)$ y mujeres $(40,7 \%)$; no preocuparse, no estresarse, no sufrir: hombres $(32,5 \%)$ y mujeres $(32,3 \%)$. En ellos es visible una actitud de resiliencia ante las dificultades, pero también, la generación de condiciones de desarrollo emocional, social, cultural y afectivo (Emerson, 1860), en el cual las personas de confianza, el proyecto de vida, el compartir con una pareja (Orgilés \& Samper, 2011), conservar un empleo que sea de agrado, y la relación con las mascotas, sirve de elemento coadyuvante en la estabilidad de las familias.

Las mascotas forman parte de la cotidianidad de las personas, son compañeros de hogar, amigos, ayudantes policiales, etc., y en muchos casos identificados como miembros activos de la familia, en otros llegan a considerarse como "hijos" por sus dueños, quienes en ocasiones presentan una mejor relación con sus mascotas que con otras personas. De acuerdo con el Diario El Espectador (2015) citando la encuesta realizada por FENALCO,

En Colombia, seis de cada diez familias cuentan con alguna mascota en su casa, siendo los perros los animales preferidos, seguidos por los gatos. Así lo revela una reciente encuesta auspiciada por la Federación Nacional de Comerciantes (FENALCO), en la cual se evidencia que el mercado de las mascotas es una gran oportunidad en "aquellas sociedades donde el crecimiento poblacional se ha vuelto lento y donde el número de hijos por mujer desciende" (p. 3).

Respecto a cifras latinoamericanas, se encontró que en México el 57\% de familias cuenta con una mascota, en Ecuador el 48\%, en Panamá 47\%, en Venezuela 38\%, mientras en Colombia es el $29 \%$. Respecto a la tipificación del perro como mascota, se encuentran presentes en el $80 \%$ de mexicanos, en el $82 \%$ de los ecuatorianos, en el $93 \%$ de panameños, en el $87 \%$ de familias venezolanas, y en el $80 \%$ de las familias colombianas, así, de acuerdo a la encuesta realizada por GfK, las familias colombianas que tienen mascotas prefieren los perros (Dinero, 2012). En gran medida la coexistencia con mascotas ayuda a las interacciones familiares, y no solo se constituyen en acompañantes, sino también en mediadores de las relaciones intrafamiliares, generando dinámicas influenciadas por la protección y el sentido de responsabilidad. Ello no quiere decir que las familias sin mascotas sean disfuncionales, sino que aquellas que eligen tener una mascota, asumen una responsabilidad adicional al cuidado de otros miembros de la familia, lo cual puede generar interacciones positivas entre sus integrantes, especialmente cuando tienen que resolver conflictos y tomarse un "tiempo íntimo" para reconsiderar sus discursos (Cruz, 2009; D'Arcy, 2011). 


\section{METODOLOGÍA}

Diseño: investigación cuantitativa, de tipo empírico-analítico y de corte transversal.

Población: investigación desarrollada en 200 familias (100 con perros y 100 sin perros) residentes en el departamento de Risaralda; la muestra no-aleatoria simple y de tipo intencionada.

Criterios de inclusión: familias con o sin canes en sus hogares y de todo tipo de familia.

Criterios de exclusión: personas que no convivan con otros individuos en su hogar.

Instrumentos: se aplica una ficha de caracterización sociodemográfica que incluye los siguientes ítems: edad, género, escolaridad, religión, etnia, tipo de vivienda, estrato; el APGAR Familiar de Gabriel Smilkstein (Alegre \& Suárez, 2006) que identifica el nivel de funcionalidad familiar de acuerdo a los siguientes intervalos: buena función familiar (18-20), disfunción familiar leve (14 - 17), disfunción familiar moderada (10-13) y disfunción familiar severa (9 o menos); el test SF-36 de calidad de vida SF-36 de Ware y Sherbourne (1992) que cuenta con 36 ítems distribuidos en 8 escalas: Funcionamiento Físico (FF); Rol Físico (RF); Dolor (D); Salud General (SG); Vitalidad (V); Funcionamiento Social (FS); Rol Emocional (RE); y Salud Mental (SM). Las 8 escalas se agrupan a su vez en salud física y salud mental; los ítems se puntúan con escalas Likert de rango variable, desde 2 valores hasta 6; su marco de referencia temporal es el momento actual y es autoaplicada.

Procedimiento: diseño y aprobación de proyecto de investigación; aprobación de las condiciones bioéticas de la investigación (consentimiento informado); firma del consentimiento informado y aplicación de instrumentos; análisis y sistematización de datos; construcción de resultados, discusión y elaboración del informe final de investigación; devolución de los resultados a las instituciones y comunidades.

\section{RESULTADOS}

La escala de funcionalidad familiar tuvo un Alfa de Cronbach de 0,914 (muy satisfactoria), mientras que, la escala: SF-36 obtuvo un Alfa de Cronbach de 0,518 (bajo). La ficha de caracterización indica que prevalecen las edades entre 22-30 años $(31,3 \%), 31-40(14,9 \%), 40-50(12,8 \%)$ y $51-65(9,2 \%)$. De los entrevistados el $29,2 \%$ fue masculino y el $70,8 \%$ fue población femenina, el $65,1 \%$ tienen religión católica y el 13,3\% cristiana; la escolaridad fue $40 \%$ bachiller, $15,1 \%$ técnico, $25,6 \%$ universitaria, 7,4\% primaria; el $46,4 \%$ vive en estrato $3,20,5 \%$ estrato $2,16,7 \%$ estrato $4,8,5 \%$ estrato 1 ; en cuanto al estado civil el $59,2 \%$ son solteros, $17,2 \%$ casados, $15,1 \%$ unión libre, $4,9 \%$ divorciados; el tipo de familia prevalente fue nuclear $(52,6 \%)$, seguida por la monoparental $(18,7 \%)$, extensa $(14,6 \%)$, y extensa compuesta (4,6\%); el 50\% de la población tiene 1 mascota (perro), el 44,1\% no tiene mascota y el 5,9\% tiene otro tipo de mascota; el 38,7\% afirma que entre todos los 
integrantes del hogar se hacen cargo de la mascota, el 11,3\% afirma que ellos mismos cuidan su mascota, el 46,4\% dicen, no conocer quién es el encargado y el 3,6\% afirman que los cuidan sus hijos o externos; en cuanto a las razones para tener una mascota el $46,7 \%$ dicen que son varias: el $16,7 \%$ para complacer un miembro de la familia, 24,4\% afirma que la mascota es el complemento de la familia y el 6,9\% por solidaridad; el 31,8\% tiene una mascota, el 14,9\% 2, el 3,3\% 3 y el 3,1\% 4 mascotas; de los encargados de las mascotas el $35,1 \%$ es estudiante, el $51,5 \%$ empleado y el $12,3 \%$ otras labores y funciones.

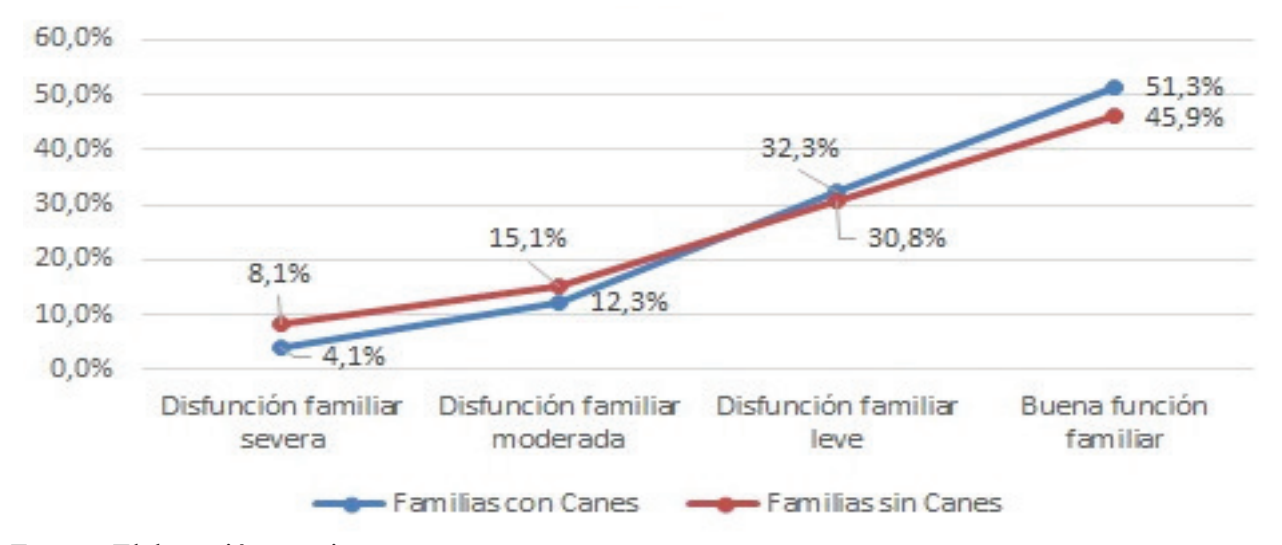

Fuente: Elaboración propia

Figura 1.

Tipo de funcionamiento familiar en familias y con y sin canes

Quienes tienen una mascota poseen una buena funcionalidad familiar $(51,3 \%)$ y quienes no (45,9\%); la disfuncionalidad familiar leve con mascota fue del 32,3\% y del 30,8\% sin mascota; la disfuncionalidad familiar moderada fue del 12,3\% con mascota y del $15,1 \%$ sin mascota; la disfuncionalidad severa fue del $4,1 \%$ con mascota y del 8,1\% sin mascota; así mismo quienes tienen algún otro tipo de mascota poseen una disfuncionalidad familiar severa del 6\% (Ilustración 1).

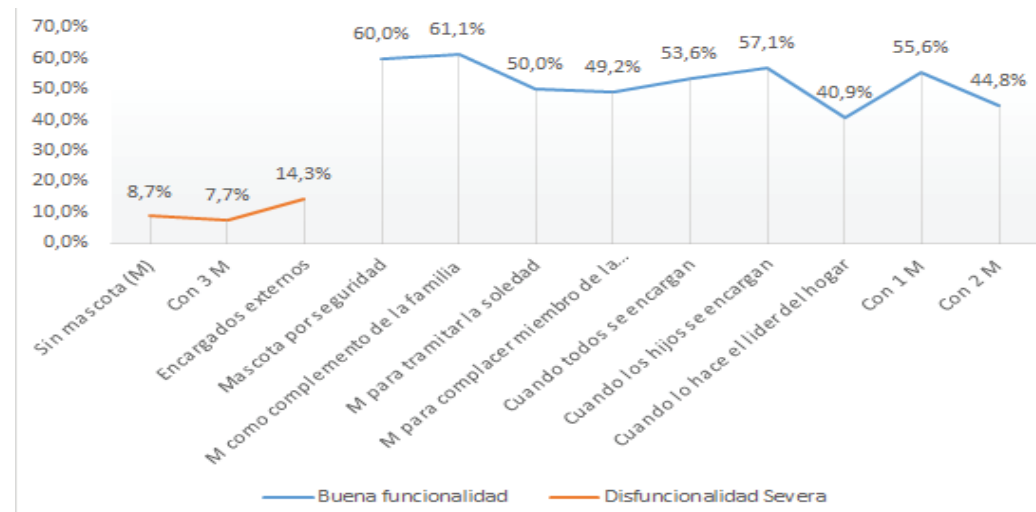

Fuente: Elaboración propia

\section{Figura 2.}

Elementos de la Funcionalidad familiar en Familias y con y sin canes. 
La buena funcionalidad familiar es mayor con una y dos mascotas $(55,6 \% ; 44,8 \%)$. La disfuncionalidad familiar severa se presentó en el $8,7 \%$ de familias sin mascotas y en el 7,7\% de familias con 3 mascotas; la buena funcionalidad familiar es mayor cuando se tiene la mascota por seguridad $(60 \%)$, cuando se cree que es el complemento de la familia $(61,1 \%)$, para facilitar la soledad $(50 \%)$, para complacer un miembro de la familia (49,2\%), existe buena funcionalidad familiar cuando todos en la familia se encargan de la mascota $(53,6 \%)$, cuando los hijos se encargan $(57,1 \%)$ y cuando lo hace el líder del hogar (40,9\%), la disfuncionalidad familiar severa se presentó en el $14,3 \%$ cuando otro externo se encarga de la mascota (Figura 2).

Tabla 1.

Elementos constitutivos de la funcionalidad familiar en familias con y sin canes.

\begin{tabular}{|c|c|c|c|c|c|c|c|}
\hline & & & Adaptación & Participación & Ganancia & Afecto & Recursos \\
\hline \multirow{4}{*}{ Mascota } & \multirow{2}{*}{$\mathrm{Si}$} & $\begin{array}{c}\text { Con } \\
\text { canes_Excelente }\end{array}$ & $52,0 \%$ & $53,3 \%$ & $51,9 \%$ & $54,4 \%$ & $50,5 \%$ \\
\hline & & $\begin{array}{c}\text { Con canes_Muy } \\
\text { Buena }\end{array}$ & $53,5 \%$ & $47,6 \%$ & $53,1 \%$ & $48,2 \%$ & $55,2 \%$ \\
\hline & \multirow{2}{*}{ No } & Sin Canes_Regular & $72,7 \%$ & $66,7 \%$ & $72,7 \%$ & $53,8 \%$ & $45,0 \%$ \\
\hline & & Sin Canes_Mala & $61,50 \%$ & $64,3 \%$ & $53,8 \%$ & $56,3 \%$ & $66,7 \%$ \\
\hline
\end{tabular}

Fuente: Elaboración propia

En la adaptación como elemento de la funcionalidad familiar, se encontró que quienes tienen mascotas poseen un nivel de adaptación excelente del $52 \%$ y muy buena del $53,5 \%$, en contraste a quienes no tienen mascotas, con una adaptación regular del $72,7 \%$; en la participación quienes tienen mascotas puntúan 53,3\% en excelente y quienes no en $66,7 \%$ en regular, en ganancia con mascota puntúan $53,1 \%$ muy buena y $51,9 \%$ excelente, en contraste a no tener mascotas con una ganancia regular del $72,7 \%$; en el afecto quienes tienen mascota $54,4 \%$ en excelente y quienes no tienen mascota 56,3\% en mala; finalmente, en el gradiente de recursos quienes tienen mascota puntúan $55,2 \%$ en muy buen acceso a ellos, y quienes no tienen mascota $66,7 \%$ en un inadecuado acceso a recursos que la familia permite o provee (Tabla 1).

\section{Tabla2.}

\section{Calidad de vida en familias con y sin canes.}

\begin{tabular}{|c|c|c|c|c|c|c|c|c|c|c|}
\hline & \multicolumn{10}{|c|}{ Mascota } \\
\hline & \multicolumn{2}{|l|}{ Si } & \multicolumn{2}{|r|}{ No } & \multicolumn{2}{|l|}{ Sí } & \multicolumn{2}{|r|}{ No } & \multicolumn{2}{|l|}{$\mathrm{Si}$} \\
\hline & Mala & Mala & Regular & Regular & Buena & Buena & $\begin{array}{c}\text { Muy } \\
\text { buena }\end{array}$ & $\begin{array}{l}\text { Muy } \\
\text { buena }\end{array}$ & Excelente & Excelente \\
\hline $\begin{array}{l}\text { Función } \\
\text { física }\end{array}$ & $0.0 \%$ & $0.0 \%$ & $0,0 \%$ & $0,0 \%$ & $0.0 \%$ & $0,0 \%$ & $39.5 \%$ & $39.5 \%$ & $50,8 \%$ & $51,7 \%$ \\
\hline Rol fisico & $71,8 \%$ & $64,0 \%$ & $0,0 \%$ & $0,0 \%$ & $0,0 \%$ & $0,0 \%$ & $0,0 \%$ & $0,0 \%$ & $0,0 \%$ & $0,0 \%$ \\
\hline $\begin{array}{l}\text { Dolor } \\
\text { corporal }\end{array}$ & $0,0 \%$ & $0,0 \%$ & $0,0 \%$ & $0,0 \%$ & $0,0 \%$ & $0,0 \%$ & $0,0 \%$ & $0,0 \%$ & $71,8 \%$ & $73,3 \%$ \\
\hline $\begin{array}{l}\text { Salud } \\
\text { general }\end{array}$ & $0,0 \%$ & $0,0 \%$ & $43,6 \%$ & $45,9 \%$ & $53,3 \%$ & $49,4 \%$ & $0,0 \%$ & $0,0 \%$ & $0,0 \%$ & $0,0 \%$ \\
\hline Vitalidad & $0,0 \%$ & $0,0 \%$ & $43,1 \%$ & $45,3 \%$ & $50,3 \%$ & $45,3 \%$ & $0,0 \%$ & $0,0 \%$ & $0,0 \%$ & $0,0 \%$ \\
\hline $\begin{array}{l}\text { Función } \\
\text { social }\end{array}$ & $0,0 \%$ & $0,0 \%$ & $19,5 \%$ & $36,0 \%$ & $72,3 \%$ & $60,5 \%$ & $0,0 \%$ & $0,0 \%$ & $0,0 \%$ & $0,0 \%$ \\
\hline $\begin{array}{l}\text { Rol } \\
\text { emocional }\end{array}$ & $0,0 \%$ & $0,0 \%$ & $0,0 \%$ & $0,0 \%$ & $0,0 \%$ & $0,0 \%$ & $0,0 \%$ & $0,0 \%$ & $69,2 \%$ & $67,4 \%$ \\
\hline
\end{tabular}

Fuente: Elaboración propia 
Con relación a la calidad de vida y sus indicadores, se tiene que la función física con mascota fue del 50,8\% excelente y $51,7 \%$ sin mascotas muy buena; $39,5 \%$ con mascota y $39,5 \%$ sin mascota; el rol físico fue malo en $71,8 \%$ con mascota y $64 \%$ sin mascota. El dolor corporal se percibe elevado en $71,8 \%$ con mascota y de $73,3 \%$ sin mascota. La salud general fue buena en $53,3 \%$ con mascota y $49,4 \%$ sin mascota, regular $43,6 \%$ con mascota y $45,9 \%$ sin mascotas. La vitalidad fue del 50,3\% con mascota y $45,3 \%$ sin mascota, regular $43,1 \%$ con mascota y $45,3 \%$ sin mascota. La función social fue buena en $72,3 \%$ con mascota y $60,5 \%$ sin mascota, y regular $19,5 \%$ con mascota y $36 \%$ sin mascota. El rol emocional fue excelente en $69,2 \%$ con mascotas y $67,4 \%$ sin mascotas. En general la salud mental y la calidad de vida fueron buenas en el $81,5 \%$ cuando se tiene mascota y en el 70,9\% sin mascota.

\section{DISCUSIÓN}

La calidad de vida es un concepto complejo, dado las múltiples condiciones, eventos y situaciones que intervienen en su percepción y sentido (Ardila, 2003). Es factible considerar que el funcionamiento familiar puede verse afectado positivamente por una mascota, resultado al que se llegó en esta investigación, especialmente en los aspectos de adaptación, participación, ganancia, afecto, generación y aprovechamiento de recursos, datos que coinciden con otras investigaciones análogas (Días \& López, 2017; Díaz, Olarte, \& Camacho, 2015; Gonzáles \& Landero, 2011). Se encontró que la calidad de vida de familias con canes suele fortalecerse en aspectos como la percepción del dolor, el funcionamiento físico y el rol emocional, puesto que se piensa que la mascota constituye un compañero que aprueba y acepta a la persona tal como es, generando a su vez estados de bienestar, percibido mejoras en la actividad física e incluso una compañía que muchas personas no logran tener (Cruz, 2009; D'Arcy, 2011; Gómez, Atehortua, \& Orozco, 2007), impactando incluso con efectos medicinales (Cruz, 2009).

Las familias sin canes también fueron funcionales, sin embargo, la disfuncionalidad familiar fue mayor (del doble) con relación a las familias con perros, esto quiere decir que las mascotas se convierten en mediadores sociales de los conflictos, y pueden con su presencia aliviar la tensión en casos de choques y crisis intrafamiliares (D’Arcy, 2011; Pedrosa, Aguado, Cafrán, Torres, \& Miró, 2016). La relación entre calidad de funcionalidad familiar es notable en los estudios sociales y constituye un indicador de ajuste social y familiar (Roca \& Riccardi, 2014; Satir, 2002), y a la vez, preparan al sistema familiar para aceptar nuevos miembros, lidiar con lo adverso y autoregularse ante estímulos estresores y conflictos emergentes, generando identidad y seguridad en sus decisiones (Minuchin, 2003). Acorde a los resultados obtenidos en esta investigación, se puede señalar que las mascotas caninas tienen la posibilidad de apuntalar dichos procesos y aumentar el estado de bienestar de las familias y sus miembros, al tiempo que mejoran la actividad grupal con otros sistemas sociofamiliares 


\section{CONCLUSIONES}

Para la calidad de vida no se encontraron diferencias significativas en la percepción entre familias con y sin canes, dado que ambas perciben calidad de vida excelente, muy buena y buena en términos similares. Es importante mencionar que en el grado malo y regular de la calidad de vida, las familias sin canes tienen un leve aumento con diferencia de las que sí tienen perros. Respecto al funcionamiento de las familias con y sin canes, se encuentra que no existen diferencias significativas elevadas a nivel estadístico entre los tipos de funcionalidad familiar, aunque las familias con canes presentan una buena funcionalidad familiar, un poco más alta que las familias que no tienen canes. En la disfuncionalidad familiar leve, ambos tipos de familia coinciden, y las disfunciones familiares moderadas y severas son un poco más altas en las familias que no tienen perros.

Respecto a la calidad de vida, aspectos como la salud mental, el rol emocional, el funcionamiento social de los miembros y percibir que se tiene vitalidad y una buena salud general, fueron mayores en las familias que tienen canes, y aunque esta diferencia no es estadísticamente elevada, sí es un indicador importante de que el convivir con mascotas fortalece la percepción de una buena calidad de vida; cabe mencionar que aquellos aspectos que tienen una significación importante en las familias con canes, son el rol emocional que la relación con la mascota provee a todos los miembros de la familia y también, un estado de salud mental elevado, dada la relación con la mascota y según las teorías, la tranquilidad que esta relación provee al núcleo familiar. Acorde al análisis de la estructura interna de la funcionalidad familiar, se encontró que para las familias con canes que tienen una buena función familiar, el perro como mascota tiene la posibilidad de generar mejores procesos adaptativos, aumentar la participación entre los miembros de la familia, lo cual produce en ellos una sensación de ganancia afectiva importante para el mantenimiento y la cohesión interna y externa de las familias. 
1. Alegre, Y., \& Suárez, M. (2006). Instrumentos de Atención a la Familia: El Familiograma y el APGAR familiar (fascículos Cadec). Rampa, 1(1), 48-57. Retrieved from http://www.idefiperu.org/RAMNRO1/N9C-PG48-CADEC Instrum Familia2A.pdf

2. Ardila, R. (2003). Calidad de vida: una definición integradora. Revista Latinoamericana de Psicología, 35(2), 161-164.

3. Cruz, C. (2009). "Mascotas: ¿Amigos medicinales?” Alternativas En Psicología, 14(20).

4. D'Arcy, Y. (2011). Las mascotas reconfortan y alivian el dolor. Nursing (Ed. Española), 29(9), 48-49. https://doi.org/10.1016/S0212-5382(11)70300-4

5. Díaz, M., \& López, P. (2017). La oxitocina en el vínculo humano-perro: revisión bibliográfica y análisis de futuras áreas de investigación. InterdIscIplInarIa, 34(1), 73-90.

6. Díaz, M., Olarte, M., \& Camacho, J. (2015). Perfiles básicos del humano compañero del perro: Una revisión teórica en antrozoología guiada por el enfoque multimodal. Rev Arg Cs Comp, 7(3).

7. Dinero, R. (2012, July). En Colombia Los prefieren perros. Revista Dinero. Retrieved from http://www.dinero.com/pais/articulo/en-colombia-los-prefierenperros/161464

8. Gómez, L., Atehortúa, C., \& Orozco, S. (2007). La influencia de las mascotas en la vida humanaף. Revista Colombiana de Ciencias Pecuarias, 20(3), 377-386.

9. Gonzáles, M., \& Landero, R. (2011). Diferencias en Estrés Percibido, Salud Mental y Física de acuerdo al Tipo de Relación Humano-Perro. Revista Colombiana de Psicología, 20(1), 75-86.

10. Ministerio de Salud y Protección Social. (2015). Encuesta Nacional En Salud Mental Colombia. Bogotá. Retrieved from http://www.visiondiweb.com/insight/ lecturas/Encuesta_Nacional_de_Salud_Mental_Tomo_I.pdf

11. Minuchin, S. (2003). Familias y terapia familiar. Barcelona: Editorial Gedisa.

12. Orgilés, M., \& Samper, M. . D. (2011). El impacto del divorcio en la calidad de vida de los niños de 8 a 12 años de edad en la provincia de Alicante. Gaceta Sanitaria, 25(6), 490-494. https://doi.org/10.1016/J.GACETA.2011.05.013

13. Pedrosa, S., Aguado, D., Cafrán, S., Torres, J., \& Miró, J. (2016). La terapia asistida con perros en el tratamiento de las personas con dolor crónico: una revisión sistemática. Rev Soc Esp Del Dolor, 24(1), 11-18.

14. Roca, M., \& Riccardi, A. (2014). Familia: Funcionalidad y efectividad como red de apoyo social en pacientes con depresión que están ingresados en el área de hospitalización de psiquiatría del hospital del IESS Dr. Teodoro Maldonado Carbo en la ciudad de Guayaquil (Spanish). Informes Psicológicos, 14(2), 81102.

15. Satir, V. (2002). Nuevas relaciones humanas en el núcleo familiar. México D.F: Editorial Pax México. 


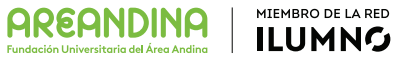

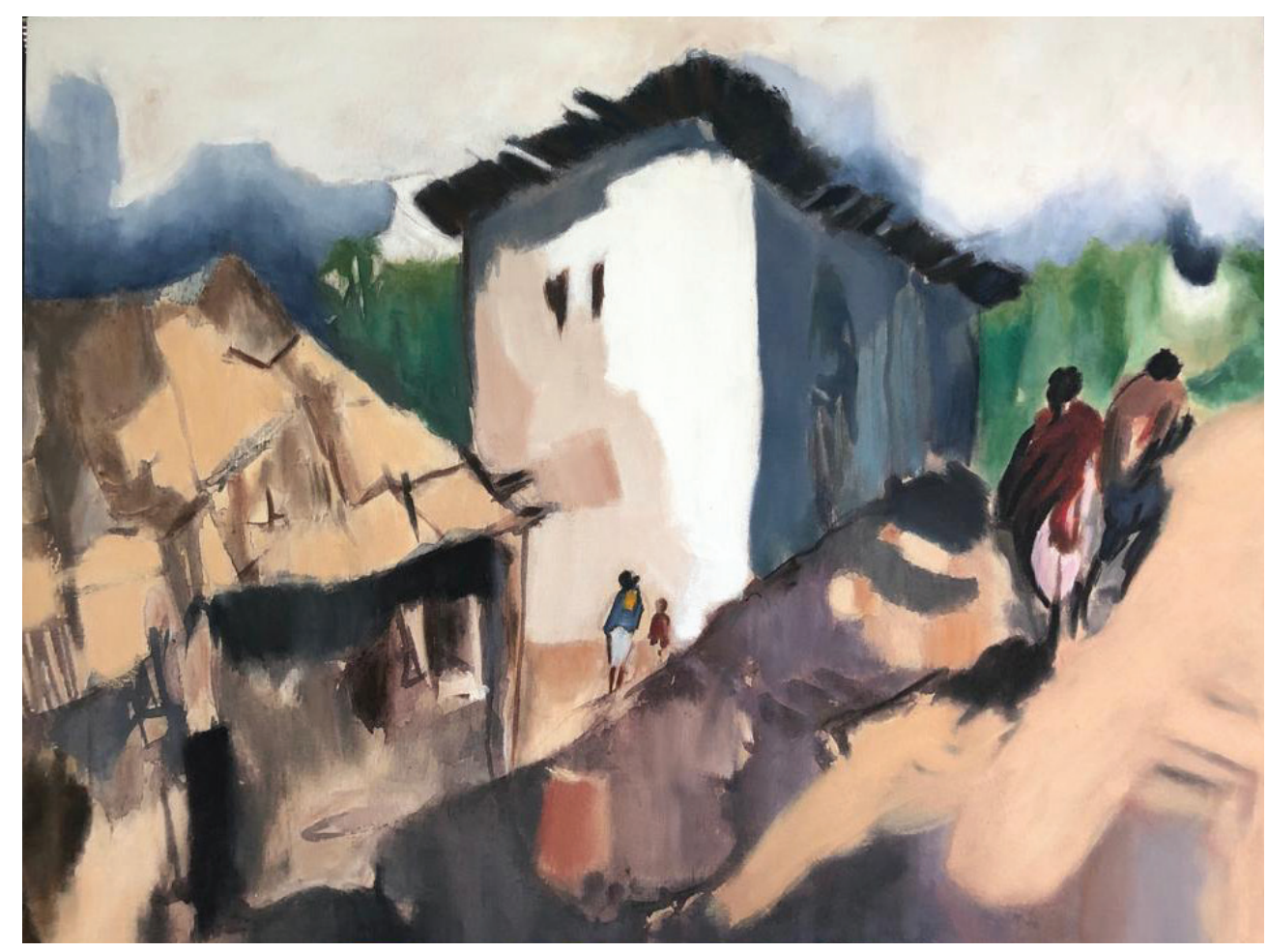

Acrílico sobre lienzo. "Paisaje colombiano”, Virginia Aristizábal Parra. 